\title{
Longitudinal dispersion control for the Keck interferometer nuller
}

Christopher D. Koresko, Bertrand P. Mennesson, Eugene Serabyn, Mark Colavita, Rachel L. Akeson, et al.

Christopher D. Koresko, Bertrand P. Mennesson, Eugene Serabyn, Mark Colavita, Rachel L. Akeson, Mark R. Swain, "Longitudinal dispersion control for the Keck interferometer nuller," Proc. SPIE 4838, Interferometry for Optical Astronomy II, (21 February 2003); doi: 10.1117/12.458032

Event: Astronomical Telescopes and Instrumentation, 2002, Waikoloa, Hawai'i, United States 


\title{
Longitudinal Dispersion Control for the Keck Interferometer Nuller
}

\author{
Chris Koresko $^{a}$, Bertrand Mennesson ${ }^{b}$, Eugene Serabyn $^{b}$, Mark Colavita $^{b}$, \\ Rachel Akeson ${ }^{a}$, and Mark Swain ${ }^{b}$ \\ ${ }^{a}$ Interferometry Science Center, Caltech, Pasadena, CA, USA \\ ${ }^{b}$ Jet Propulsion Laboratory, Caltech, Pasadena, CA, USA
}

\begin{abstract}
The control of longitudinal dispersion, which determines the position of the null fringe as a function of wavelength, is central to the problem of producing deep broadband interferometric nulls. The dispersion is the sum of terms due to environmental factors such as the dry-air and water-vapor atmospheric seeing, the unbalanced air column due to the unequal delay-line paths between the telescopes the combiner, and to the distance from the central fringe. The difference between an achromatic nuller and a normal constructive combiner operating at its first (chromatic) null can be thought of as an added longitudinal dispersion, which for the case of the Keck Interferometer is smaller than the environmental terms. We demonstrate that the sum of these effects can be adequately compensated by an appropriate thickness of ZnSe combined with an additional achromatic pathlength. The Keck Interferometer nulling combiners take advantage of this result. They are intrinsically constructive combiners made to produce achromatic nulls by inserting a ZnSe dispersion corrector into each of the four input beams. We describe the null fringe stabilization control algorithm and present calculations of the required loop bandwidth and precision. A potentially important advantage of the present approach is that the system will be able to function as either a destructive or constructive combiner, depending on the value of a single control-loop parameter (the target fringe phase).
\end{abstract}

Keywords: Interferometry, Astronomy, Dispersion

\section{INTRODUCTION}

The modified Mach-Zehnder (MMZ) pupil-plane interferometric beam combiner design ${ }^{1}$ used for the Keck Interferometer Nuller adds the fields in two input beams without producing a relative phase shift, so that the combined output reaches its maximum intensity when the phases of the input wavefronts are identical. This design was chosen for its high degree of intrinsic symmetry, which reduces the likelihood that its performance will be compromised by unbalanced reflectivity, transmissivity, or polarization effects in its optical components. A nulling beam combiner (a "nuller") differs from the constructive combiner in that the nuller produces destructive rather than constructive interference when the input wavefront phases are matched. A nuller is well suited to addressing a certain class of astronomical problems in which one wishes to detect a faint source (e.g., a dust disk or a planet) very close to a much brighter one (e.g., the star which the faint source orbits). A spatiallyfiltered pupil-plane interferometer whose inputs are fed by beams coming from separate telescope apertures has a response pattern on the sky which consists of a single-aperture response pattern (typically similar to an Airy pattern) modulated by a set of narrow fringes. For a nuller, the central fringe is destructive. If the bright source falls within the central dark fringe ("null") while the faint source lies at least partly outside it, then the detected light comes disproportionately from the faint source.

For the simple case of a monochromatic system, a nuller can be implemented by adding a half-wavelength delay to one of the inputs of a constructive combiner. This is equivalent to shifting the fringe pattern on the sky by half a wavelength, such that the center changes from bright to dark. For polychromatic light, the position of the dark fringe on the sky is wavelength-dependent, and this limits the depth of the null. The null is described as "chromatic". In most astronomical applications, the brightness of the faint companions is low enough to require a substantial optical bandpass in order to collect enough photons to achieve a useful signal-to-noise ratio 
in a reasonable time. The intrinsic chromaticity in such a broad bandpass severely limits the contrast of the fringe pattern.

What is needed is a way to make the null achromatic, i.e., to cause the position of the null to become independent of wavelength. In principle, the cleanest way to do this is by rotating the polarization vectors of the inputs to a constructive combiner to make them opposed to each other, so that they interfere destructively when the wavefronts are in phase ${ }^{2}$. This makes the central fringe (where the difference in the optical pathlengths of the input beams vanishes) change from bright to dark. In principle, this approach makes the central null perfectly achromatic and allows for arbitrarily deep nulls even for a large optical bandpass. The alternative approach is to pass the input beams through dispersive elements which produce a relative wavefront delay that approximately compensates for the wavelength dependence of the null produced by a constructive combiner. The null depth which can be achieved this way is a function the accuracy of the compensation.

For a ground-based nulling interferometer which uses delay lines at ambient atmospheric pressure to compensate for the unequal wavefront phases at the telescope apertures, the chromaticity associated with moving half a wavelength away from the central fringe of a constructive beam combiner is not the dominant chromatic term. The unequal pathlengths through the humid air between the telescopes and the beam combiner can be much larger. In addition, the column densities of water vapor above the telescopes can differ enough to contribute significantly. The presence of these atmospheric terms makes the field-flip approach much less attractive than it would otherwise be, since the field flip can in general address only a small part of the chromaticity problem, leaving the remainder to be handled with dispersive components. It is simpler to use a single set of dispersion correctors to compensate for the intrinsic chromaticity of a constructive combiner at null in addition to the much larger atmospheric terms.

For this reason, the dispersive compensation approach has been chosen for the Keck Interferometer Nuller. We note that dispersion in ZnSe has previously been chosen to produce the achromatic half-wavelength phase shift in the nulling beam combiner for the Multiple Mirror Telescope (MMT) and the Large Binocular Telescope $(\mathrm{LBT})^{3}$. This paper presents the results of an analytical calculation which demonstrates that readily available materials can dispersively compensate the null fringe chromaticity with sufficient precision to produce the target null depth of $10^{-4}$ over a useful bandpass between 10.0 and $12.5 \mu \mathrm{m}$. We first consider the various intrinsic and atmospheric terms separately and in combination, and derive a general formula for computing the optimum thickness of glass to compensate them. We then examine some subtleties in the problem. Finally, we consider the properties of the time-variability of the water vapor differential column, which we refer to as "water seeing," and examine the possibility of measuring it with a near-infrared fringe tracker.

\section{CORRECTING THE RELATIVE WAVEFRONT PHASE}

The wavelength-dependent relative wavefront phase $\phi(\lambda)$ at wavelength $\lambda$ for a two-input beam combiner is

$$
\phi(\lambda)=\frac{2 \pi}{\lambda}\left[\Delta_{v a c}+\Delta_{a i r} n_{a i r}+\Delta_{c o r} n_{c o r}\right]
$$

The value of $\Delta_{v a c}$ is the unbalanced pathlength through vacuum, which for the Keck interferometer occurs above the atmosphere, and $\Delta_{a i r}$ is the unbalanced distance through air, which is mainly due to the path added in the ambient-pressure delay lines. The unbalanced pathlength through the dispersion correctors is $\Delta_{\text {cor }}$. The wavelength-dependent refractive indices of air and the dispersion corrector material are given by $n_{\text {air }}$ and $n_{c o r}$, respectively.

Producing a deep null requires making $\phi(\lambda)$ close to $(2 m+1) \pi$, where $m$ is an arbitrary integer, for all $\lambda$ in the bandpass. The desired system null depth (the ratio of the signal at the destructive valley to the signal at the constructive peak, measured for an idealized star with zero angular size) for the Keck Nuller is $10^{-4}$. This requires the phase to be correct to within approximately 0.02 radians, in the absence of other error terms. In the case of the Keck Nuller, the range of $\lambda$ is determined mostly by the science goals and by the transmission of Earth's atmosphere, while $\Delta_{v a c}$ is set by the interferometer baseline and the position of the star in the sky. The index $n_{\text {air }}$ of the air at the site of the instrument cannot be controlled with useful precision, and the choice 
of dispersion corrector material fixes $n_{\text {cor }}$. This leaves $\Delta_{a i r}$ and $\Delta_{\text {cor }}$ as free parameters which can be adjusted to optimize $\phi(\lambda)$. Note that these unbalanced pathlengths can be made either positive or negative.

Because the relative phase is the sum of a set of independent contributions, and because it is measured at a finite number of discrete wavelengths $\lambda_{i}$ determined by the spectral resolution of the nuller camera, the problem of computing the best $\left\{\Delta_{\text {air }}, \Delta_{\text {cor }}\right\}$ can be expressed in matrix form. Define matrix $\mathbf{A}$, whose columns are the partial derivatives of the relative phase $\phi\left(\lambda_{i}\right)$ with respect to $\Delta_{a i r}$ and $\Delta_{c o r}$, as follows:

$$
\mathbf{A}=2 \pi\left(\begin{array}{cc}
\lambda_{1}{ }^{-1} n_{\text {air }}\left(\lambda_{1}\right) & \lambda_{1}{ }^{-1} n_{\text {cor }}\left(\lambda_{1}\right) \\
\lambda_{2}{ }^{-1} n_{\text {air }}\left(\lambda_{2}\right) & \lambda_{2}{ }^{-1} n_{\text {cor }}\left(\lambda_{2}\right) \\
\vdots & \vdots \\
\lambda_{N}{ }^{-1} n_{\text {air }}\left(\lambda_{N}\right) & \lambda_{N}{ }^{-1} n_{c o r}\left(\lambda_{N}\right)
\end{array}\right)
$$

Let $\mathbf{B}$ be the 2-vector containing the adjustments $\delta_{a i r}$ and $\delta_{c o r}$ to be made to $\Delta_{a i r}$ and $\Delta_{c o r}$ :

$$
\delta \mathbf{B}=\left(\begin{array}{c}
\delta_{\text {air }} \\
\delta_{\text {cor }}
\end{array}\right)
$$

and define the vector $\delta \phi$, which describes the change in the relative phase due to $\mathbf{B}$, as

$$
\delta \phi=\left(\begin{array}{c}
\delta \phi\left(\lambda_{1}\right) \\
\vdots \\
\delta \phi\left(\lambda_{N}\right)
\end{array}\right)=2 \pi\left(\begin{array}{c}
\lambda_{1}^{-1}\left[n_{a i r}\left(\lambda_{1}\right) \delta_{a i r}+n_{c o r}\left(\lambda_{1}\right) \delta_{c o r}\right] \\
\vdots \\
\lambda_{N}{ }^{-1}\left[n_{a i r}\left(\lambda_{N}\right) \delta_{a i r}+n_{c o r}\left(\lambda_{N}\right) \delta_{c o r}\right]
\end{array}\right)=\mathbf{A} \delta \mathbf{B}
$$

Solving this to find $\mathbf{B}$ for a given $\delta \phi$ (in the least-squared sense, assuming that the number of measured wavelengths is larger than 2) gives

$$
\delta \mathbf{B}=\mathbf{S} \delta \phi
$$

where

$$
\mathbf{S}=\left(\mathbf{A}^{\mathrm{T}} \mathbf{A}\right)^{-1} \mathbf{A}^{\mathrm{T}}
$$

The T superscript denotes a transposition. The above formulation gives the values of $\delta_{\text {air }}$ and $\delta_{\text {cor }}$ which produce the best approximation, in the least-squared sense, of a given $\delta \phi$. Producing an achromatic null requires $\delta \phi=$ $(2 m+1) \pi-\phi\left(\lambda_{i}\right)$. In practice, the accuracy with which this value can be approximated depends on the choice of $m$. So one would test each of a series of $m$ values and choose the one which permits the smallest phase error.

It is worth noting here that one can just as easily tune the delay lines and dispersion corrector to produce a constructive combiner instead of a nuller. This requires nothing beyond changing the value of $\delta \phi$ to $2 m \pi-\phi\left(\lambda_{i}\right)$ in the above computation.

\section{CHROMATIC TERMS}

We consider three sources of chromaticity in the wavelength band between 10.0 and $12.5 \mu \mathrm{m}$ : unbalanced pathlengths through dry air (meaning all molecules in ambient air other than water vapor), water vapor itself, and the intrinsic dispersion due to the use of a constructive beam combiner operating half a wavelength from the central fringe. It will be seen that the water vapor is the dominant source of chromaticity.

The chromatic terms due to dry air and water vapor, and the intrinsic chromaticity due to the use of a constructive combiner as a nuller, are plotted in Figures 1-3. The residual phase due to the sum of these terms, after correction with a ZnSe dispersion corrector, is plotted in Figure 5. In each plot, an achromatic relative pathlength offset has been added to minimize the mean-squared value in each curve. 


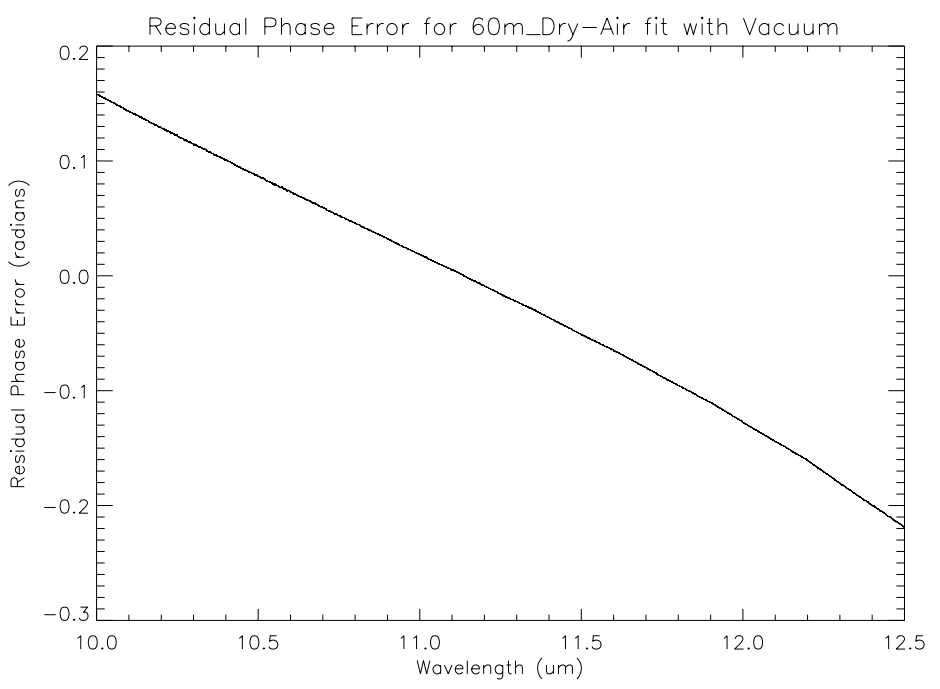

Figure 1. Phase error produced by $60 \mathrm{~m}$ of dry air at a pressure of $0.6 \mathrm{~atm}$. The curve can be fit fairly well with dispersive solids such as ZnSe.

\subsection{Chromaticity of Dry Air and Water Vapor}

The refractive indices of dry air and water vapor have been computed by summing the contributions from individual absorption lines using a code due to R. Hill (unpublished). Hill has done extensive previous work on computation of the refractive indices of gases, including water vapor ${ }^{4}$, in the infrared. Here we present the results in a form appropriate for the Keck Nuller. The unbalanced pathlengths for dry air and water are assumed to be $60 \mathrm{~m}$, and the ambient pressure is 0.6 atmospheres. The volume density of water molecules is taken to be $10^{17} \mathrm{~cm}^{-3}$, which corresponds to a relative humidity of $32 \%$ at a temperature of $10 \mathrm{C}$, typical of conditions in the beam-combining lab.

The assumed unbalanced path through dry air and water vapor produce the phase error curves plotted in Figures 1 and 2. It can be seen that both the overall shape and the magnitude of the curves are significantly different, and that the water is by far the most important. Beyond the larger amplitude of the smoothly-varying component, water exhibits a number of absorption lines in the Keck nuller's bandpass, and these produce highfrequency structure in the phase curve which becomes important once the smoothly-varying component is suppressed by the dispersion corrector.

\subsection{Intrinsic Chromaticity of a Constructive Combiner used as a Nuller}

If there is an achromatic difference of $\frac{\lambda_{0}}{2}$ in the pathlengths for the input beams for an intrinsically constructive combiner, then it will act as a nuller at wavelength $\lambda_{0}$. However, at nearby wavelengths the cancellation of the input beams will be imperfect. As a function of wavelength $\lambda$ the relative phase is

$$
\phi(\lambda)=\left(\frac{\lambda_{0}}{\lambda}-1\right) \pi
$$

which varies from -0.31 radians at $12.5 \mu \mathrm{m}$ to +0.39 radians at $10.0 \mu \mathrm{m}$ if $\lambda_{0}$ is taken as $11.25 \mu \mathrm{m}$. The intrinsic chromaticity is plotted in Figure 3.

\subsection{ZnSe Dispersion Correctors}

In Figure 5 we show the phase due to a combination of dry air, water, intrinsic chromaticity, and a suitable thickness of $\mathrm{ZnSe}$ used as a dispersion corrector, for a particular set of conditions chosen to represent something 


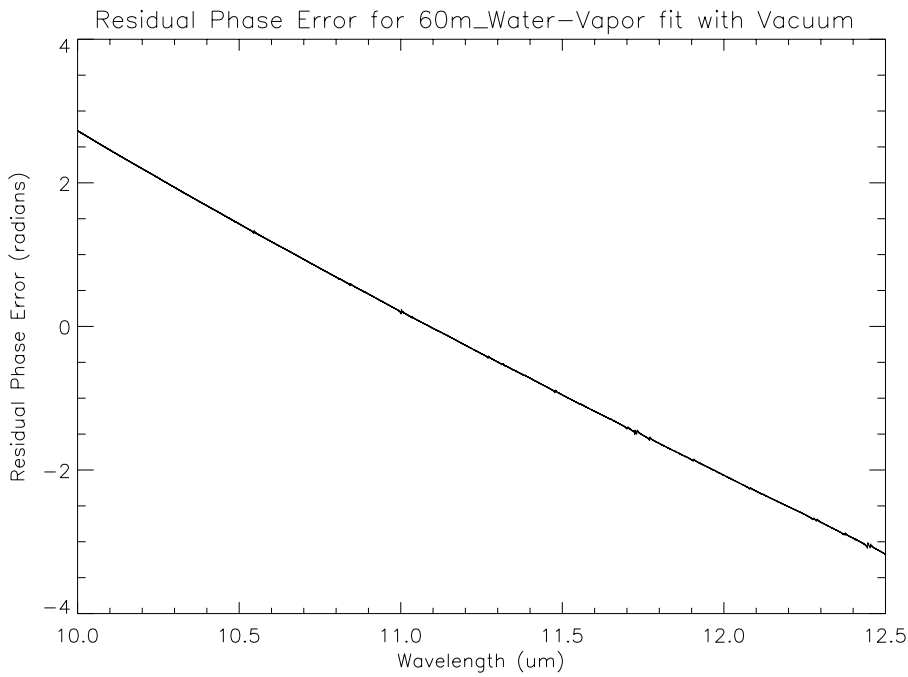

Figure 2. Phase error produced by $60 \mathrm{~m}$ of water vapor with a density of $10^{17}$ molecules $\mathrm{cm}^{-3}$, corresponding to a relative humidity of $32 \%$ at $10 \mathrm{C}$. Several strong lines are just visible in the plot. They will be significant contributors to the phase residuals once the smoothly-varying term has been compensated with glass. Note that the overall dispersion for water is an order of magnitude larger at these wavelengths than that for dry air for the assumed conditions.

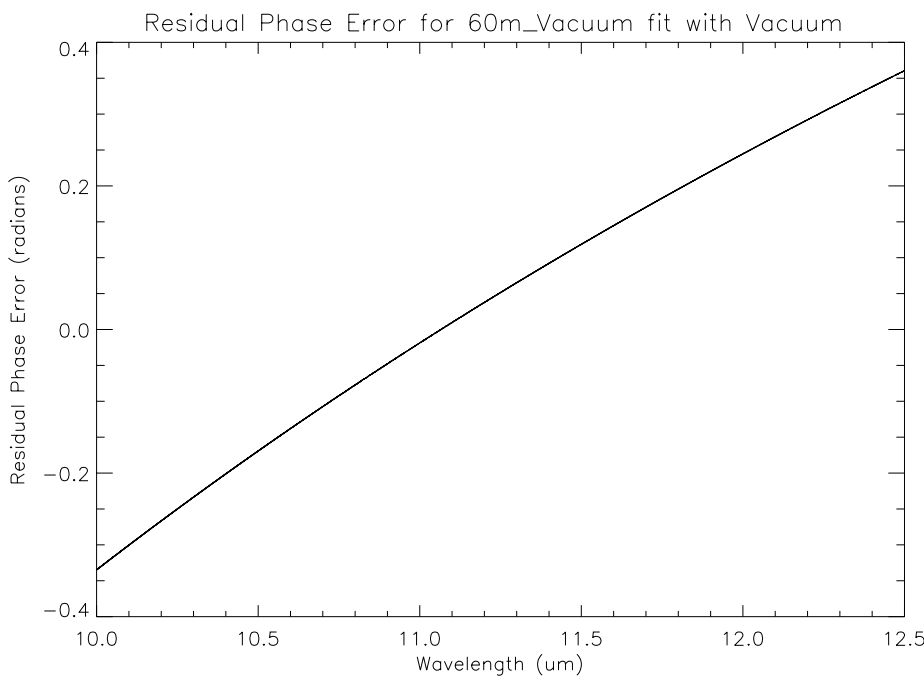

Figure 3. Phase error produced by intrinsic chromaticity due to operation of a beam combiner half a wavelength from the central fringe. 


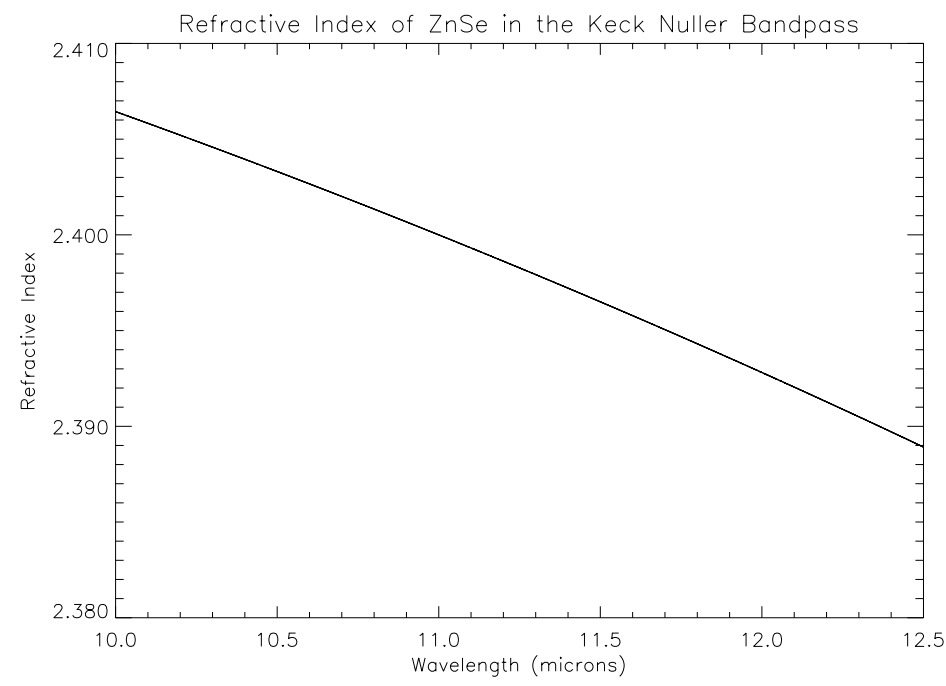

Figure 4. Refractive index of ZnSe in the bandpass of the Keck Interferometer Nuller.

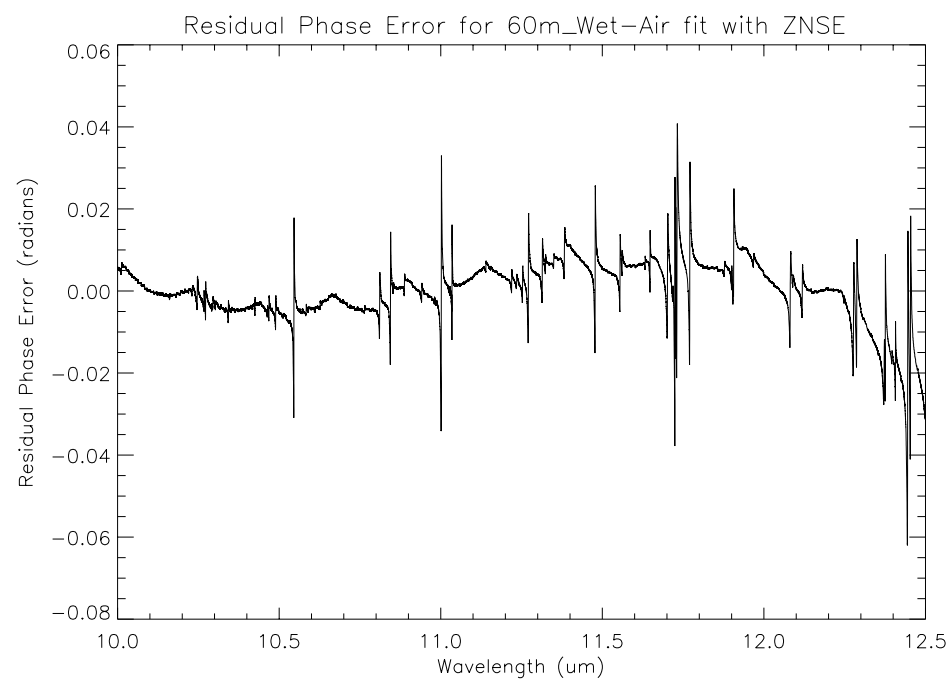

Figure 5. Residual phase error after correcting the intrinsic chromaticity and $60 \mathrm{~m}$ of wet air (i.e., dry air plus water vapor) with $0.6 \mathrm{~mm}$ of $\mathrm{ZnSe}$. The RMS residual is only 0.007 radians. 
Table 1. Specifications for the Keck nuller ADCs. Each ADC is formed of a pair of 6-deg wedges, and has 4 anti-reflection coated glass-air interfaces.

\begin{tabular}{|c|c|}
\hline Wedge angle & $6 \mathrm{deg} \pm 20 \mathrm{arcsec}$ \\
\hline Initial wedge parallelism & 12 arcmin \\
\hline Stroke range & $\pm 10 \mathrm{~mm}$ \\
\hline Stroke step & $10 \mu \mathrm{m}$ \\
\hline Displacement accuracy (linear) & $\pm 70 \mu \mathrm{m}$ \\
\hline Displacement accuracy (tilt) & $\pm 11 \mathrm{arcsec}$ \\
\hline Translation speed & $100 \mu \mathrm{m} / \mathrm{s}$ \\
\hline AR coating (@10.6 $\mu \mathrm{m})$ & $\mathrm{R}<0.5 \%$ \\
\hline AR coating (across medium band) & $\mathrm{R}<3 \%$ \\
\hline
\end{tabular}

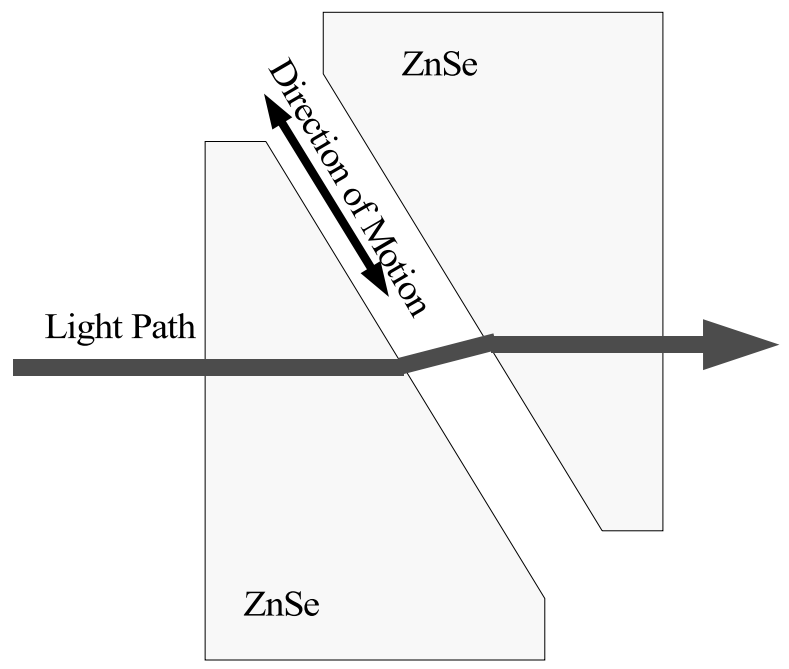

Figure 6. Schematic of a single dispersion corrector showing the pair of wedges and the path the light takes through them. The actual wedge angle is shallower than what is shown, and the distance between the wedges is larger.

close to the worst case. The relative pathlength through ZnSe for the assumed conditions is $0.57 \mathrm{~mm}$. In practice, this thickness will need to be adjusted to accommodate the changing unbalanced airpath, the humidity in the beam-combining lab, and the differential water vapor column above the telescopes. This will be accomplished by inserting into each of the nuller's input beams a pair of right-angle ZnSe wedges, as shown schematically in Figure 6. One of the wedges is made to slide in a direction parallel to its long face in order to adjust the ZnSe pathlength seen by a beam passing through the system parallel to its right-angle faces. In this configuration, a beam passing through the pair of wedges can be made to undergo no deviation and only a fixed displacement. A pair of dispersion correctors which are part of the Keck nuller are shown in Figure 7. Their design specifications are given in Table 1.

\section{EFFECT OF WATER VAPOR SEEING}

It is anticipated that the most challenging dispersion-control problems faced by the Keck Interferometer Nuller will be caused by the changing differential water-vapor column density above the two Keck telescopes. These 


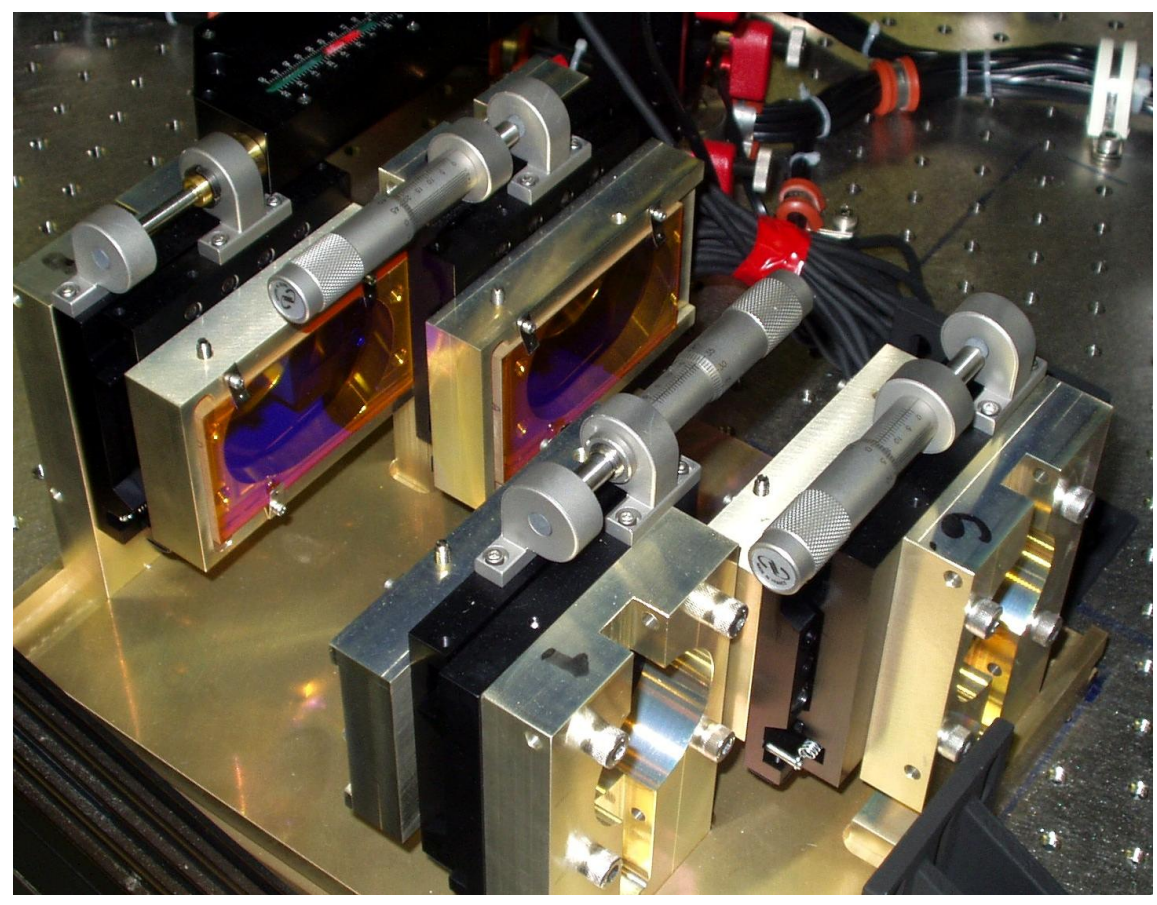

Figure 7. Photograph of a pair of dispersion correctors as used in the Keck Nuller. The fixed ZnSe wedges are in the mounts labeled " 1 " and " 6 " in the lower right of the image, and their actively-adjustable counterparts are in the upper left.

changes have significant power on timescales as short as $1 \mathrm{sec}$ or less. They produce two deleterious effects:

N-band fringe motion: The Keck Nuller relies on feedforward from the K-band fringe tracker to provide information about the N-band fringe position on short timescales.

K-N dispersion: Relative motion between the K-band and N-band fringes. Changes in the differential water column will cause the offset between the K-band and N-band fringes to change. This will have to be dealt with either by periodically measuring the N-band fringe position, or by monitoring the changing differential water column.

N-band dispersion: Nonzero derivative of N-band fringe position with wavelength, over and above that caused by the unbalanced basement airpath and the intrinsic chromaticity. As the wind blows patches of varying water column density across the telescope apertures, the dispersion correctors and delay lines will have to be adjusted to compensate.

This section presents a calculation of the magnitude of the latter two effects on relevant timescales.

\subsection{Temporal Power Spectrum of the Water Seeing}

Measurements of the fluctuations in the phase measured by a two-element radio interferometer on Mauna Kea with a $100 \mathrm{~m}$ baseline and operating at $12 \mathrm{GHz}$ are available in the literature ${ }^{5}$. The source was a beacon tone from a geostationary satellite. The RMS fluctuation in the residual phase was calculated for each 15-minute interval after removal of a linear time dependence attributed to the motion of the satellite. The fluctuations are somewhat sensitive both to the hour of the day and to the season, with winter nights having the smallest 
fluctuations. 50th-percentile nights have relative pathlength fluctuations of $\sim 75 \mu \mathrm{m}$ RMS at $12 \mathrm{GHz}$, measured over 15-minute intervals, for much of the year. Given that the ratio of the precipitable water vapor column (PWV) to the optical pathlength it produces at radio wavelengths is $1 / 6$, this corresponds to fluctuations in the PWV of $13 \mu \mathrm{m}$, in the column mass density of $1.3 \times 10^{-3} \mathrm{gm} \mathrm{cm}^{-2}$, and in the column number density of $\sigma_{\Sigma, 15}=4 \times 10^{19} \mathrm{~cm}^{-2}$. Note that the above implicitly assumes that the refractivity of water is proportional to its number density and independent of the overall air pressure. Because the refractivity at radio wavelengths is due to the wings of pressure-broadened lines, it is roughly proportional to the product of the water density and the air pressure. If that scaling also applies in the infrared, then the $\sigma_{\phi, 15}$ values in the tables presented below will be too high by a factor of roughly 1.7 for Mauna Kea's ambient pressure of $0.6 \mathrm{~atm}$.

The RMS fluctuations due to a noise signal, after filtering it to a given bandwidth, are proportional to the square root of the integral of the power spectrum over that bandwidth:

$$
\sigma\left(f_{1}, f_{2}\right)=\sigma\left(f_{\min }, f_{\max }\right) \sqrt{\frac{\int_{f_{1}}^{f_{2}} P(f) d f}{\int_{\max } f_{\min } P(f) d f}}
$$

where $\sigma\left(f_{1}, f_{2}\right)$ is the RMS fluctuation in the signal in a band of frequencies in the range $\left(f_{1}, f_{2}\right)$, and $\left(f_{\min }, f_{\max }\right)$ defines the range for which the power spectrum $P(f)$ of the phase fluctuations were originally measured. Thus the functional form of the power spectrum, combined with the value of the RMS measured for a broad range of timescales and the tolerance on the N-band phase error, can be used to derive the minimum bandwidth for the servo loop which tracks the fluctuations. Note that in practice this will involve an extrapolation of the power spectrum to frequencies beyond those measured by M94.

M94 finds that the power spectrum of the phase fluctuations for "typical" conditions follows a broken power law, with the power proportional to $\left(f / f_{c}\right)^{-0.5}$ for $f<f_{c}$, and to $\left(f / f_{c}\right)^{-2.5}$ for higher frequencies, and the corner frequency $f_{c}=0.01 \mathrm{~Hz}$. Plugging in the value of the RMS column density fluctuation derived above, assuming that $f_{1}>f_{c}$, taking $f_{2} \rightarrow \infty$, taking $\left(f_{\min }, f_{\max }\right)$ to define the range of frequencies sampled by M94, and integrating, gives

$$
\sigma\left(f_{1}\right)=0.018 \sigma_{15}\left(f_{1} / \mathrm{Hz}\right)^{-0.75}
$$

where $\sigma_{15}$ and $\sigma\left(f_{1}\right)$ are the RMS fluctuations of any quantity proportional to the unbalanced water vapor column, measured for the whole 15 -minute period and for frequencies above $f_{1}$, respectively.

The phase offset produced by a water column number density $\Sigma$ at wavelength $\lambda$, at which the refractivity of water is $N_{\lambda, 17}$ for a number density of $10^{17} \mathrm{~cm}^{-3}$, is

$$
\Delta \phi(\lambda)=\frac{2 \pi}{\lambda} \frac{N_{\lambda, 17}}{10^{17} \mathrm{~cm}^{-3}} \Sigma
$$

The Hill program gives $N_{\lambda, 17}$ over a range of infrared wavelengths. With these values and $\sigma_{\Sigma, 15}=4 \times 10^{19} \mathrm{~cm}^{-2}$, we find $\sigma_{\phi, 15}$, the 15-minute RMS fluctuation in the phase due to water vapor seeing, at several relevant wavelengths. Equation 9 then gives the RMS residual fluctuation after filtering by a servo loop which eliminates frequencies below $1 \mathrm{~Hz}$. The results are presented in the following table:

\begin{tabular}{cccc}
$\lambda(\mu \mathrm{m})$ & $N_{\lambda, 17}$ & $\sigma_{\phi, 15}(\mathrm{rad})$ & $\sigma_{\phi}(1 \mathrm{~Hz})(\mathrm{rad})$ \\
\hline & & & \\
2.1 & $9.035 \times 10^{-7}$ & 10.8 & 0.19 \\
2.3 & $8.962 \times 10^{-7}$ & 9.8 & 0.18 \\
10.0 & $6.797 \times 10^{-7}$ & 1.7 & 0.030 \\
12.5 & $5.022 \times 10^{-7}$ & 1.0 & 0.018
\end{tabular}


One can use these results to compute the RMS fluctuation of the dispersion $\sigma_{\text {Disp }, 15}$ across each of these bands:

\begin{tabular}{ccc} 
Band $(\mu \mathrm{m})$ & $\sigma_{\text {Disp }, 15}(\mathrm{rad} / \mu \mathrm{m})$ & $\sigma_{\text {Disp }}(1 \mathrm{~Hz})(\mathrm{rad} / \mu \mathrm{m})$ \\
\hline & & 0.09 \\
$2.1-2.3$ & 5.1 & 0.005
\end{tabular}

Between the $\mathrm{K}$ and $\mathrm{N}$ bands the dispersion is best computed from the difference in the OPD changes induced by the water seeing fluctuations. The RMS fluctuation in the phase in the $\mathrm{N}$ band when the K-band fringe is perfectly tracked is given by

$$
\sigma_{\phi_{N-K, 15}}=\frac{2 \pi}{\lambda_{N}} \frac{N_{\lambda_{N}, 17}-N_{\lambda_{K}, 17}}{10^{17} \mathrm{~cm}^{-3}}
$$

Plugging in the numbers gives $\sigma_{\phi_{N-K, 15}}=0.7$ radians. Using Equation 9 to scale this for a $1 \mathrm{~Hz}$ cutoff frequency gives 0.013 radians.

The final step is to solve for the servo loop bandwidth required to suppress water seeing induced chromaticity fluctuations, both within the $\mathrm{N}$ band and between the $\mathrm{N}$ and $\mathrm{K}$ bands, to the required level of 0.01 radians. An RMS fluctuation of the dispersion across the $\mathrm{N}$ band of $0.3 \mathrm{rad} \mu \mathrm{m}^{-1}$, and the requirement that this be suppressed to give a total of 0.01 radians across the band between 10.0 and $12.5 \mu \mathrm{m}$, requires the servo to

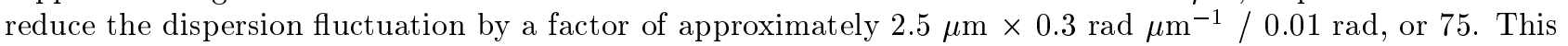
requires $f_{1}=1.3 \mathrm{~Hz}$. Taking the uncorrected N-band phase fluctuations expected for perfect K-band fringe tracking $\left(\sigma_{\phi_{N}, 15}=0.7\right.$ radians over a 15 -minute period), and solving for $\sigma\left(f_{1}\right)=0.01$ radians, gives $f_{1}=1.3$ $\mathrm{Hz}$. Thus the bandwidth requirement for the monitoring of the water vapor differential column is $1.3 \mathrm{~Hz}$ for both dispersion terms.

\section{CONCLUSIONS}

Solving the dispersion problem is fundamental to conducting nulling long-baseline interferometric observations of celestial sources from the ground. We have demonstrated that the form and magnitude of the chromatic terms due to dry air, water vapor, and the intrinsic chromaticity associated with the use of a constructive beam combiner operating half a wavelength from its central fringe as a nuller, can be compensated sufficiently well with a $\mathrm{ZnSe}$ dispersion corrector to produce the required null depth for the Keck Interferometer nuller. In light of this result, we have abandoned the "field flip" which would have made the beam combination intrinsically destructive for equal input optical pathlengths, but which would not compensate for the larger chromatic terms due to dispersion in the unbalanced airpaths. This represents a small but significant simplification to the design of the instrument.

The most challenging aspect of the chromaticity problem is likely to be the rapid variability of the dispersion due to the water seeing. Based on published radio interferometer measurements of the power spectrum of the column density fluctuations at Mauna Kea, we have estimated the servo loop bandwidth required to track and compensate the fluctuations well enough to maintain the required overall precision for the compensated phase. The bandwidth turns out to be of the order of $1 \mathrm{~Hz}$ for typical conditions. Calculations indicate that measurements of the differential water vapor column can be made with sufficient accuracy at this speed using the dispersed fringe data produced by the K-band fringe tracker.

\section{Acknowledgments}

Funding was provided by NASA through the Keck Interferometer and Terrestrial Planet Finder projects. The research presented here was conducted at the Jet Propulsion Laboratory, California Institute of Technology, under contract with the National Aeronautics and Space Administration. 


\section{REFERENCES}

1. E. Serabyn \& M.M. Colavita, "Fully Symmetric Nulling Beam Combiners ", Applied Optics, 40, 1668, 2001.

2. E. Serabyn, these proceedings.

3. P.M. Hinz, J.R.P. Angel, W.F. Hoffman, D.W. McCarthy, P.C. McGuire, M. Cheselka, J.L. Hora, \& N.J. Woolf, "Imaging circumstellar environments with a nulling interferometer", Nature 395, 251, 1998.

4. R.J. Hill \& R.S. Lawrence, "Refractive index of water vapor in infrared windows", Infrared Phys, 26, 371, 1986.

5. Colin R. Masson, "Atmospheric effects and calibrations", ASP Conference Series, 59, M. Ishiguro \& Wm. J. Welch (eds.) 1994. 